\title{
The Persistent Infection of Tick Cells by Hazara orthonairovirus Is Mediated by Virus-Derived DNA Forms ${ }^{+}$
}

\author{
Maria Vittoria Salvati ${ }^{1}$, Claudia Del Vecchio ${ }^{1}$, Lesley Bell-Sakyi ${ }^{2}$, Cristina Parolin ${ }^{1}$, \\ Arianna Calistri ${ }^{1}$, Giorgio Palù ${ }^{1}$, Ali Mirazimi ${ }^{3,4,5}$ and Cristiano Salata ${ }^{1, *}$ \\ 1 Department of Molecular Medicine, University of Padova, 35121 Padova, Italy; \\ mariavittoria.salvati@phd.unipd.it (M.V.S.); claudia.delvecchio@unipd.it (C.D.V.); \\ cristina.parolin@unipd.it (C.P.); arianna.calistri@unipd.it (A.C.); giorgio.palu@unipd.it (G.P.) \\ 2 Department of Infection and Microbiome, Institute of Infection, Veterinary and Ecological Sciences, \\ University of Liverpool, Liverpool L3 5RF, UK; L.Bell-Sakyi@liverpool.ac.uk \\ 3 Department of Microbiology, Public Health Agency of Sweden, SE-171 82 Solna, Sweden; \\ Ali.Mirazimi@ki.se \\ 4 Department of Laboratory Medicine, Karolinska University Hospital and KI, \\ SE-14186 Huddinge Stockholm, Sweden \\ 5 National Veterinary Institute, SE-756 51 Uppsala, Sweden \\ * Correspondence: cristiano.salata@unipd.it \\ + Presented at Viruses 2020-Novel Concepts in Virology, Barcelona, Spain, 5-7 February 2020.
}

Published: 6 July 2020

\begin{abstract}
Introduction: Crimean-Congo hemorrhagic fever (CCHF) is a severe disease of humans caused by CCHF orthonairovirus (CCHFV), a class 4 pathogen. Hyalomma ticks are the viral reservoir, and they represent the main vector. CCHFV can be transmitted to its hosts during tick blood feeding. We have previously shown that CCHFV can persistently infect Hyalomma-derived tick cell lines without any cytopathic effect. However, the mechanism allowing for the establishment of a persistent viral infection in ticks is still unknown. It has been recently reported that Hazara orthonairovirus (HAZV) can be used as a BSL-2 model virus instead of CCHFV to study viral/vector interaction. The aim of our study is to elucidate the mechanism that allows establishment of persistent CCHFV infection in ticks using HAZV as a model. Methods: We used classical and molecular methods applied to virology to characterize the establishment of persistent HAZV infection in two Hyalomma anatolicum-derived cell lines-HAE/CTVM8 and HAE/CTVM9. Results: As for CCHFV, we showed that HAZV persistently infects tick cells without any sign of cytopathic effect and that infected cells can be cultured for more than one year. The persistent infection is characterized by a low viral titer compared to the initial time points. Interestingly, short viralderived DNA forms (vDNAs) start to be detected in parallel with the beginning of viral replication and are maintained in persistently-infected cells. Experiments with the antiretroviral drug AZT suggest that vDNAs are produced by retrotranscriptase activity. Furthermore, we collected evidence that vDNAs are not integrated and seem to be involved in the downregulation of viral replication by promoting cell survival. Conclusion: vDNA synthesis might represent a strategy to control the replication of RNA viruses in ticks, as recently demonstrated in insects, allowing for persistent infection of virus vectors.
\end{abstract}


Keywords: Hazara orthonairovirus; tick cell line; viral-derived DNA forms; persistent infection; tick-borne disease; Crimean-Congo hemorrhagic fever orthonairovirus; Hyalomma; reversetranscriptase

(C) 2020 by the authors. Licensee MDPI, Basel, Switzerland. This article is an open access article distributed under the terms and conditions of the Creative Commons Attribution (CC BY) license (http://creativecommons.org/licenses/by/4.0/). 